\title{
Human seasonal influenza under COVID-19 and the potential consequences of influenza lineage elimination
}

\section{Vijaykrishna Dhanasekaran ( $\nabla$ veej@hku.hk)}

The University of Hong Kong https://orcid.org/0000-0003-3293-6279

\section{Sheena Sullivan}

Doherty Institute https://orcid.org/0000-0002-0856-0294

\section{Kimberly Edwards}

The University of Hong Kong

\section{Ruopeng Xie}

The University of Hong Kong

\section{Arseniy Khvorov}

The Peter Doherty Institute for Infection and Immunity, The University of Melbourne and Royal Melbourne Hospital

\section{Sophie Valkenburg}

The University of Hong Kong https://orcid.org/0000-0002-0768-9926

\section{Benjamin Cowling}

University of Hong Kong https://orcid.org/0000-0002-6297-7154

\section{lan Barr}

WHO Collaboratng Centre for Reference and Research on Influenza

\section{Article}

Keywords: seasonal influenza, short- and long-term evolutionary dynamics, influenza lineage elimination

Posted Date: September 14th, 2021

DOl: https://doi.org/10.21203/rs.3.rs-850533/v1

License: (c) (i) This work is licensed under a Creative Commons Attribution 4.0 International License. Read Full License

Version of Record: A version of this preprint was published at Nature Communications on March 31st, 2022. See the published version at https://doi.org/10.1038/s41467-022-29402-5. 


\section{Human seasonal influenza under COVID-19 and the potential consequences of influenza lineage elimination}

Vijaykrishna Dhanasekaran, ${ }^{1,2 *}$ Sheena Sullivan, ${ }^{3}$ Kimberly M. Edwards, ${ }^{1,2}$ Ruopeng Xie, ${ }^{1,2}$ Arseniy Khvorov, ${ }^{3}$ Sophie A. Valkenburg, ${ }^{1,2}$ Benjamin J. Cowling, ${ }^{1}$ Ian G. Barr ${ }^{3}$

${ }^{1}$ School of Public Health, LKS Faculty of Medicine, The University of Hong Kong, Hong Kong, China

${ }^{2}$ HKU-Pasteur Research Pole, School of Public Health, LKS Faculty of Medicine, The University of Hong Kong, Hong Kong, China

${ }^{3}$ WHO Collaborating Centre for Reference and Research on Influenza, Royal Melbourne Hospital, at the Peter Doherty Institute for Infection and Immunity, Melbourne, Victoria 3000, Australia

*Corresponding author:

Vijaykrishna Dhanasekaran

School of Public Health, LKS Faculty of Medicine, The University of Hong Kong, Hong Kong, China

email: veej@hku.hk 
1 Annual epidemics of seasonal influenza cause hundreds of thousands of deaths, high

2 levels of morbidity, and substantial economic loss. Yet, global influenza circulation has

3 been heavily suppressed by public health measures and travel restrictions since the onset of the COVID-19 pandemic. Notably, the influenza B/Yamagata lineage has not been conclusively detected since April 2020, and A(H3N2), A(H1N1), and B/Victoria viruses circulate with considerably less genetic diversity. Travel restrictions have largely confined regional outbreaks of $\mathrm{A}(\mathrm{H} 3 \mathrm{~N} 2)$ to South and Southeast Asia, B/Victoria epidemics in China, and $\mathbf{A}(\mathrm{H} 1 \mathrm{N1})$ in West Africa. Seasonal influenza transmission lineages continue to perish globally, except in select hotspots, which will likely seed future epidemics. Waning population immunity and sporadic case detection will further challenge influenza vaccine strain selection and epidemic control. We offer perspective on the potential short- and long-term evolutionary dynamics of seasonal influenza and discuss potential consequences and mitigation strategies as global travel gradually returns to pre-pandemic levels.

Human seasonal influenza evolution has been well studied, ${ }^{1}$ and although vaccines that confer partial protection have been available for many decades, ${ }^{2}$ seasonal influenza epidemics remain a substantial public health burden. Prior to COVID-19 emergence, influenza caused 290,000650,000 deaths annually ${ }^{3}$ and an estimated loss of over $\$ 11$ billion to the United States economy alone. ${ }^{4}$

Seasonal influenza viruses evolve to evade pre-existing immunity and gain competitive advantage via surface protein mutations which yield new antigenic variants. ${ }^{1}$ Natural selection acts on a global scale due to rapid and widespread global circulation. ${ }^{5}$ This effectively eliminates previously dominant antigenic variants and results in limited circulation of antigenically similar viruses within each subtype/lineage at a given point in time. However, the pace of antigenic selection varies over time for influenza A virus (IAV) subtypes and influenza $\mathrm{B}$ virus (IBV) lineages. This confounds vaccine strain selection, which relies on the prediction of antigenic evolution. ${ }^{6}$ To facilitate bi-annual selection of candidate vaccine viruses, the WHO Global Influenza Surveillance and Response System (GISRS) coordinates influenza surveillance from 138 National Influenza Centers (NICs) and diagnostic and reference quadrivalent, with either three or four representative strains including IAV subtypes $A(H 1 N 1)$ and $\mathrm{A}(\mathrm{H} 3 \mathrm{~N} 2)$ and either one or both IBV lineages, B/Victoria and B/Yamagata. 
Seasonal influenza viruses exhibit stronger seasonal cycles in temperate zones, with surges of infections in winter. Seasonal trends are weaker in tropical zones, with increased circulation evident in both the rainy season due to increased humidity and in cooler, drier months. ${ }^{8,9}$ Seasonal cycles are maintained through continuous reintroduction from tropical regions and opposing hemispheres, causing local transmission chains to emerge and perish in community settings. ${ }^{5,10,11}$ Transmission chains arising from a single introduction (transmission lineages) dissipate at a greater frequency outside of peak seasonal circulation, although some may persist from one season to the next. ${ }^{5,12}$ In tropical regions, influenza viruses exhibit more complex multi-peak dynamics, impacted by patterns of global circulation and evolution. ${ }^{13}$ The interplay between the different seasonal influenza virus subtypes and lineages varies temporally and geographically, leading to significant variation in population immunity to each influenza virus.

Analysis of global sequence data has shown that (i) tropical and subtropical regions in Asia sustain transmission lineages for a longer duration than temperate regions, providing more opportunities for antigenic drift, ${ }^{5}$ and (ii) A(H3N2) lineages do not generally persist between seasons in temperate regions but are reseeded annually, whereas transmission lineages of $\mathrm{A}(\mathrm{H} 1 \mathrm{N1}), \mathrm{B} /$ Yamagata, and B/Victoria can circulate for several years without reseeding from tropical regions. Population density and regional interconnectedness play an important role in maintaining viral metapopulations. ${ }^{11,17}$ However, the genetic and antigenic diversity of seasonal influenza has been severely impacted by dramatic changes in global migration and travel since the onset of the COVID-19 pandemic in March 2020 (Figure 1).

Since April 2020, most countries have seen historically low seasonal influenza virus circulation $^{18,19}$ attributable to non-pharmaceutical interventions (NPIs) such as travel restrictions, quarantine on arrival, social distancing, school and workplace closures, mask wearing, surface disinfection, and enhanced hand hygiene. NPIs have similarly disrupted the circulation of other common respiratory viruses such as respiratory syncytial virus and human metapneumovirus ${ }^{20-23}$ by limiting opportunities for reintroduction and local transmission. Prolonged suppression of seasonal influenza virus circulation compounded by challenges in vaccine access will reduce population immunity and increase severity of future influenza virus epidemics. In general, population immunity to influenza wanes over two to seven years due to antigenic evolution of the surface proteins. At the individual level, circulating antibodies decline over six months, ${ }^{14}$ and the half-life of T-cells for cellular responses lasts eight to 14 years. ${ }^{15}$ Accumulation of susceptible individuals during milder seasons results in more intense 

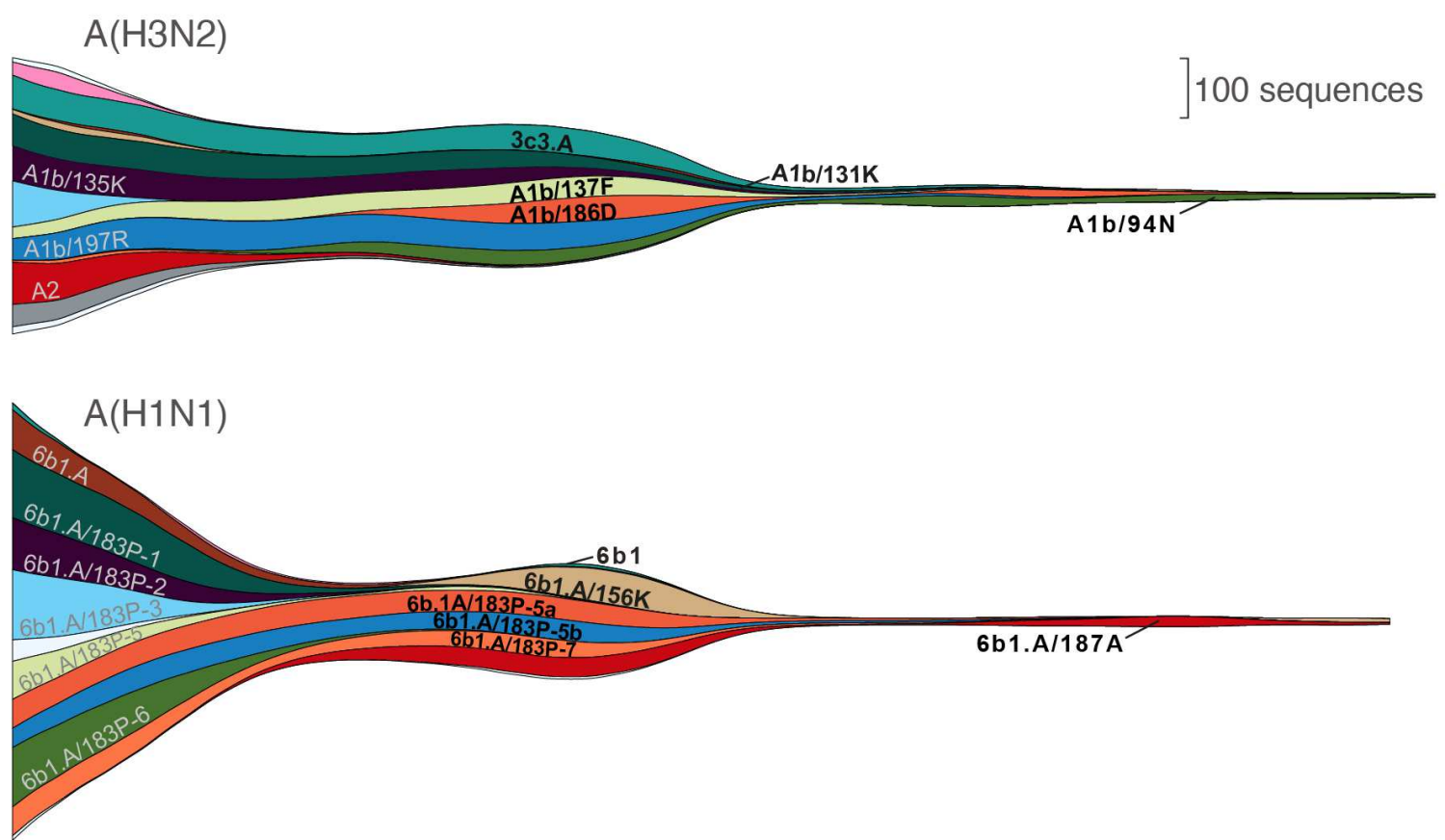

B/Victoria

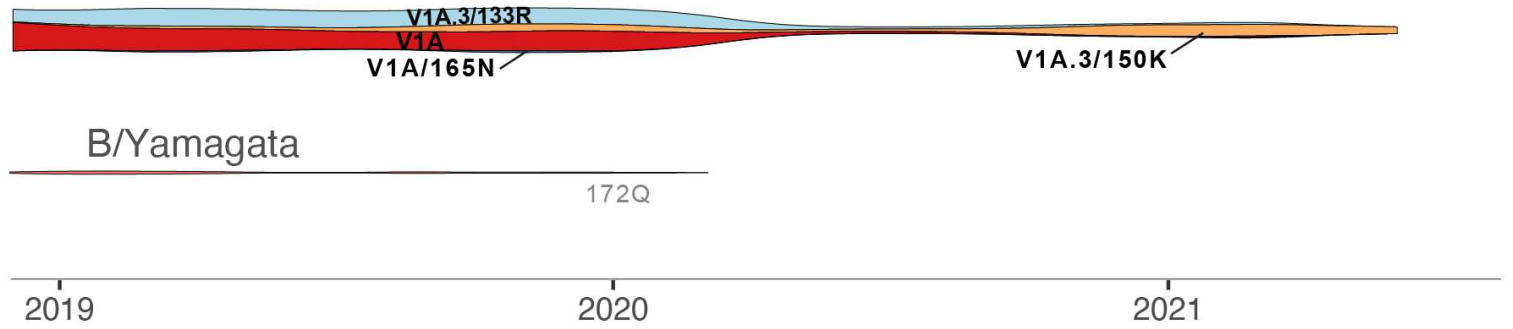

Figure 1. Streamgraph showing temporal changes in influenza lineage circulation. Lineage prevalence was estimated using sample collection dates of all sequences submitted to the Global Initiative for Sharing All Influenza Data (GISAID) from December 2018 to August 2021. Lineages detected since April 2020 are labelled in black; lineages that have not been detected since April 2020 are labelled in gray. subsequent seasonal epidemics. ${ }^{16}$ The consequences may be most dire for children, as childhood influenza infections impact patterns of susceptibility and circulation in subsequent years. ${ }^{23}$ Epidemiological studies, corroborated by multiple modeling and immunological studies ${ }^{24,25}$ show lifelong immune memory to first childhood influenza infection confers lifelong homosubtypic protection at the cost of heterosubtypic protection. Recently, Vieira et $a l .{ }^{24}$ examined historical dominant subtype frequencies in New Zealand using statistical modeling and showed additional protection against B/Yamagata for individuals imprinted with this lineage. Prolonged suppression of seasonal influenza circulation during the 2020s will lead 
to greater susceptibility in this birth cohort due to lack of exposure by infection and reduced vaccine access.

As COVID-19 vaccination rates increase in the coming months, the use of NPIs to limit transmission will gradually decline. Domestic and international travel will eventually return to pre-pandemic levels, ${ }^{26}$ enabling a resurgence of influenza virus circulation. Through phylogenetic analysis of available influenza sequence data and case reports submitted to WHO GISRS we consider the short- and long-term implications of COVID-19 control measures on the epidemiology and evolution of seasonal influenza viruses and discuss possible strategies to mitigate future influenza epidemics.

\section{Results}

\section{A global reduction in seasonal influenza virus case detection}

Analysis of the GISRS FluNet database ${ }^{7}$ to August 1 $1^{\text {st }}, 2021$ shows an unprecedented global reduction in seasonal influenza cases since the beginning of the COVID-19 pandemic in March 2020 (Figure 2). Influenza testing capacity declined during the initial stages of the pandemic amid the high demand for SARS-CoV-2 testing. Nevertheless, many countries continued or resumed influenza testing and reporting by mid-2020, ${ }^{20,27,28}$ and the dramatic decline in influenza virus detection cannot be explained by this transient reduction in laboratory testing. During the 2017/2018 to 2019/2020 Northern Hemisphere winter seasons, the number of influenza positive cases peaked around 40,000 to 60,000 per week. In early February 2020, cases in the Northern Hemisphere fell from a peak of approximately 50,000 cases per week to less than 100 cases per week in May 2020 and remained below 100 weekly cases until September 2020, a 99.8\% reduction (Figure 2). During the first half of 2021, case numbers increased marginally to 200 to 400 cases per week. Similarly, in the Southern Hemisphere, activity during the 2017-2019 seasons peaked between 1,500-3,500 positive specimens per week, but the 2020 season was notably absent and the expected rise in seasonal influenza cases has yet to occur in 2021. Remarkably, $<12$ influenza positive cases per week were reported from May 2020 to July 2021 in the Southern Hemisphere (Figure 2). 
A
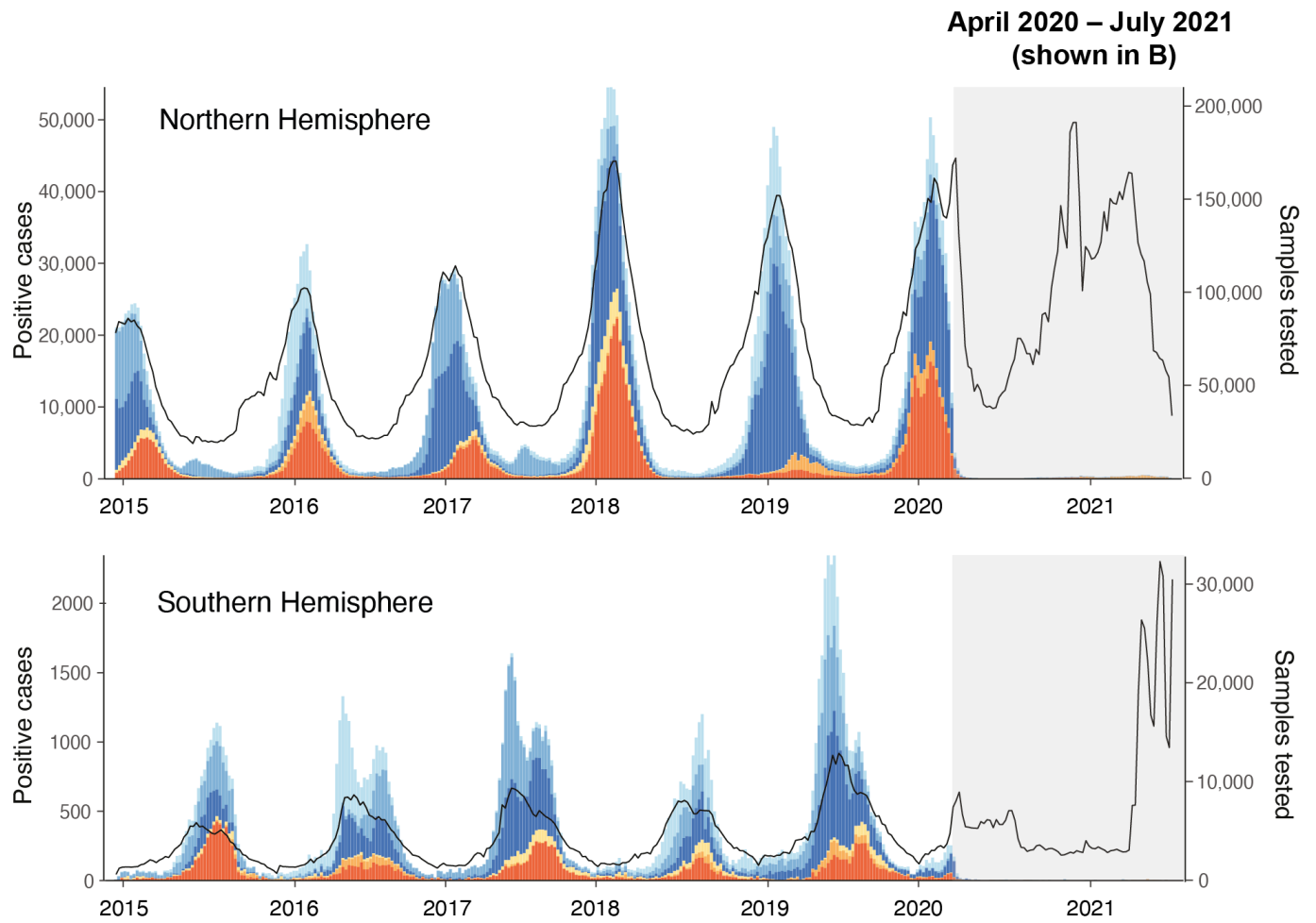

B
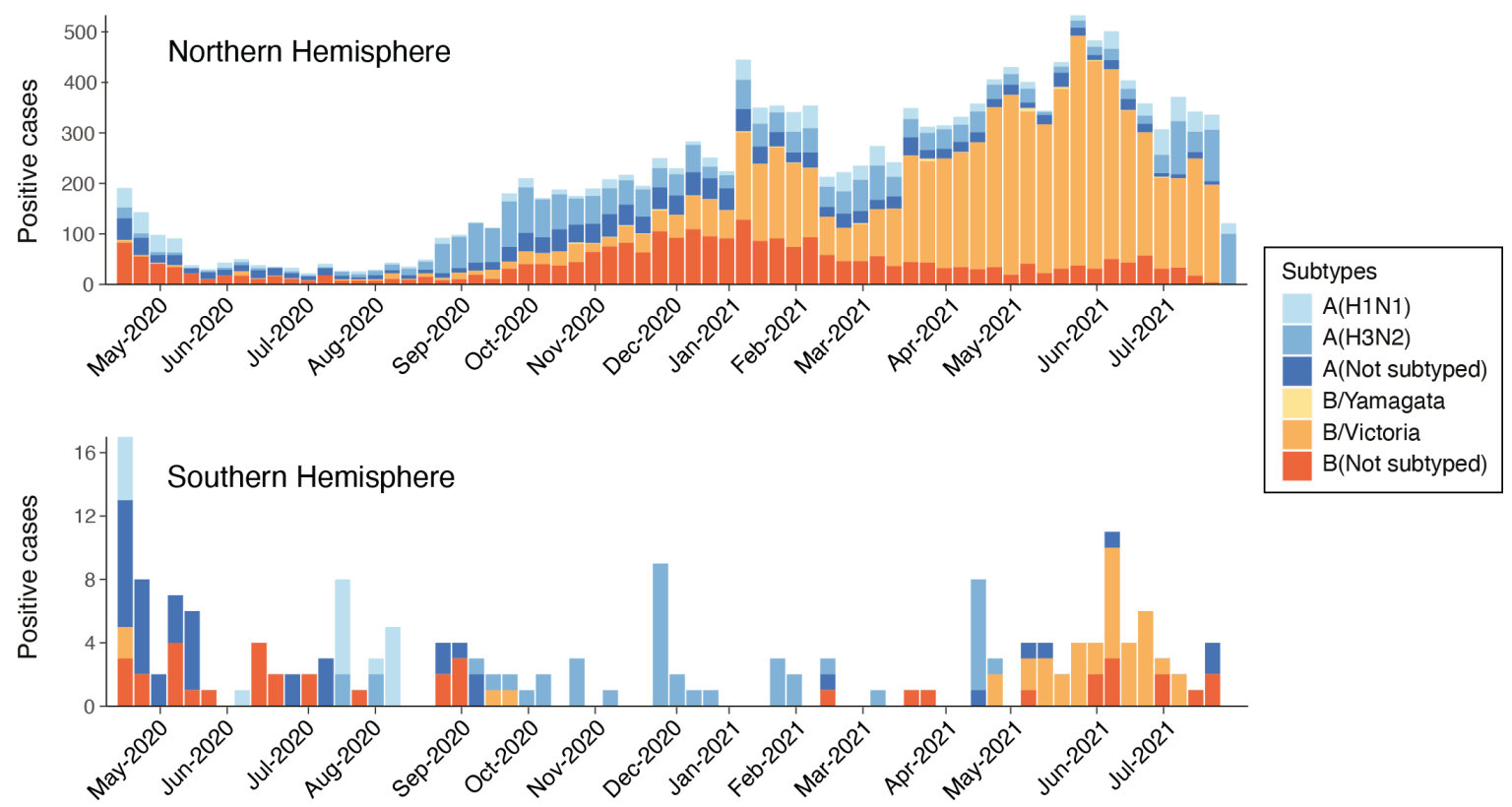

Figure 2. Epidemiological trends of seasonal influenza viruses. A. Time series comparing

FluNet data on seasonal influenza activity in the Northern and Southern Hemispheres from 2015 to July 2021, with the COVID-19 pandemic shaded in gray. Stacked bar chart (left-hand y-axis) represents the number of influenza-positive cases per week colored by subtype. Black trend-line (right hand y-axis) shows the number of specimens tested per week. B. Magnified view of the gray-shaded bar charts in A showing influenza-positive specimens from April 2020 to July 2021. Note: y-axis scales differ in each panel. 
Co-circulation of diverse $\mathrm{A}(\mathrm{H} 3 \mathrm{~N} 2)$ and $\mathrm{B} /$ Victoria viruses and antigenic drift within some cocirculating clades have necessitated frequent updates to the vaccine strain components in recent years. Since emergence in 1968, A(H3N2) viruses have, on average, evolved distinct antigenic variants every three to seven years with rapid elimination of previous antigenic variants. ${ }^{29,30}$ However, leading up to the COVID-19 pandemic, a major A(H3N2) genetic bottleneck had not occurred for a number of years ${ }^{31}$ (Figures 1,3). The continued circulation of an A(H3N2) clade 3c3.A, a lineage which dates back to 2013, has been implicated in reduced production of neutralizing antibodies in adults with childhood exposure to $\mathrm{A}(\mathrm{H} 3 \mathrm{~N} 2),{ }^{25}$ and it has been hypothesized that further accumulation of antigenic changes may result in $\mathrm{A}(\mathrm{H} 3 \mathrm{~N} 2)$ divergence. ${ }^{32}$ Like A(H3N2), the genetic diversity of B/Victoria expanded from 2015 to 2018, with seven subclades co-circulating prior to the COVID-19 pandemic, albeit with less antigenic diversity than $\mathrm{A}(\mathrm{H} 3 \mathrm{~N} 2)$. In contrast, $\mathrm{A}(\mathrm{H} 1 \mathrm{~N} 1)$ has shown slower antigenic drift, with $6 \mathrm{~b} 1 / 183 \mathrm{P}-5 \mathrm{a}$ as the dominant $\mathrm{A}(\mathrm{H} 1 \mathrm{~N} 1)$ clade amid a number of antigenically related subclades, and B/Yamagata viruses have exhibited weak antigenic selection in recent years, further reducing their prevalence over time. ${ }^{33}$

From April 2020 through July 2021, only 2,521 influenza A(H3N2) virus cases were reported from 57 countries in FluNet (Extended Data Table 1), with outbreaks apparent in West Africa (Côte d'Ivoire $(\mathrm{n}=123)$ and Senegal $(\mathrm{n}=119))$, South Asia (Bangladesh $(\mathrm{n}=209)$, India $(\mathrm{n}=$ 455), Pakistan $(\mathrm{n}=162)$, and Nepal $(\mathrm{n}=107))$, and Southeast Asia (Cambodia $(\mathrm{n}=108)$, Laos $(\mathrm{n}=268)$, and Vietnam $(\mathrm{n}=162))$. Only $590 \mathrm{~A}(\mathrm{H} 3 \mathrm{~N} 2)$ sequences from this 16-month period were deposited in GISAID, a $>97 \%$ reduction in $\mathrm{A}(\mathrm{H} 3 \mathrm{~N} 2)$ sequence data globally in comparison to the previous 16 months. Of the eight $\mathrm{A}(\mathrm{H} 3 \mathrm{~N} 2)$ subclades that circulated during while 3c3.A, A1b/94N, A1b/186D, A1b/131K, and A1b/137F continue to circulate in 2021 (Figures 1,3). 


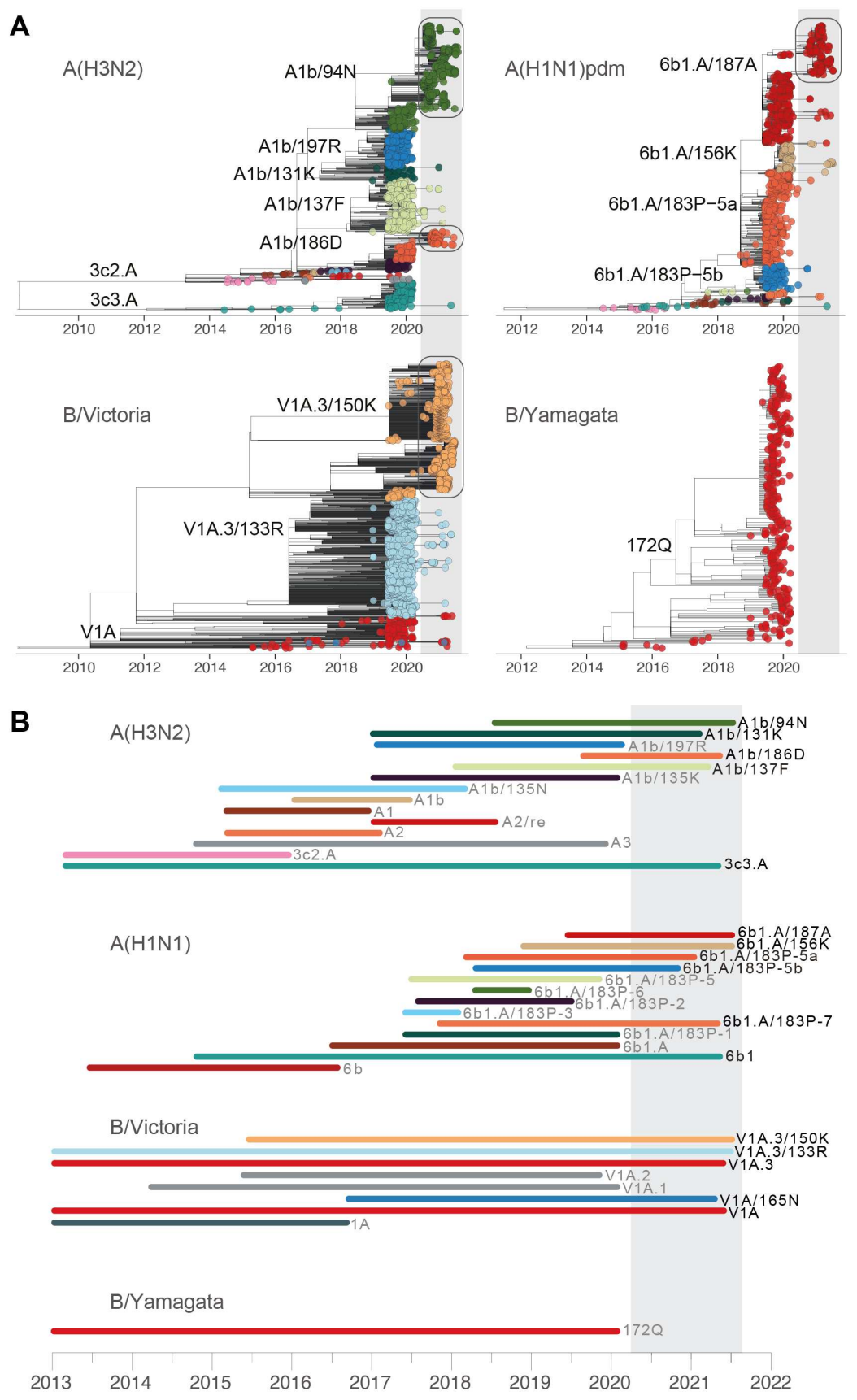

emergence. A. Evolutionary relationships and divergence times of HA genes inferred using

142 maximum likelihood (see Online Methods). Tips are colored by WHO clade designations.

143 Phylogenetic trees of major clades that continued to circulate during 2020/2021 (marked in A) are shown in Extended Data Figures 1-4, including A(H3N2) clades A1b/94N and A1b/186D; $\mathrm{A}(\mathrm{H} 1 \mathrm{~N} 1)$ clade 6b1.A/187A lineages in West Africa; and B/Victoria lineages from several regions around the world. B. Timeline of recently circulating seasonal influenza virus HA

147 clades from mean estimated divergence time to most recent sequence. Time since the onset of the COVID-19 pandemic is shaded in gray. 
Maximum likelihood phylogenetic analysis of seasonal influenza virus hemagglutinin (HA) sequences since SARS-CoV-2 emergence illustrates the circulation of geographically and genetically distinct $\mathrm{A}(\mathrm{H} 3 \mathrm{~N} 2)$ outbreaks in parts of West Africa, South Asia, and Southeast Asia (Figure 4 and Extended Data Table 2). Clade A1b/137F viruses were detected sporadically in Bangladesh, Laos, New Zealand, United Arab Emirates, and Yunnan province in China (Figure 4 and Extended Data Table 2). A(H3N2) clade A1b/186D was detected primarily in West Africa (Extended Data Figure 1), while clade A1b/94N was detected mainly in across Asia and Oceania (Extended Data Figure 2). The phylogeny of clade A1b/94N reveals six related clusters that originated independently prior to March 2020. First detected in Cambodia, one lineage circulated in the Greater Mekong sub-region of Southeast Asia from July to February 2020. A second distinct A1b/94N lineage was detected in the Australian Northern Territory from individuals returning to Australia and in quarantine during February-March 2021 and from neighboring Timor-Leste during July 2020-March 2021, suggesting regional circulation during 2020/2021 (Extended Data Figure 2). Three other A1b/94N lineages, represented by only one or two identical HA sequences, were detected in Japan on March $2^{\text {nd }}$, 2021, Bangladesh on September $28^{\text {th }}, 2021$, and Côte d'Ivoire on November $24^{\text {th }}, 2021$. While most $\mathrm{A} 1 \mathrm{~b} / 94 \mathrm{~N}$ lineages have been regionally contained, one $\mathrm{A} 1 \mathrm{~b} / 94 \mathrm{~N}$ lineage with common ancestry dating back to 2019 in South Asia has been detected in India, Bangladesh, United Arab Emirates, Australia, Kenya, Singapore, the United States, and several European countries during 2020 and early 2021(Figures 3A,4, Extended Data Figure 2 and Extended Data Table 2).

Few A(H1N1) cases have been detected since April 2020 (Figure 2), mainly in Ghana ( $\mathrm{n}=$ 235), Togo $(n=226)$, the United States $(n=170)$, and Russia $(n=165)$ (Extended Data Table 1). Nevertheless, the 254 available sequences in GISAID reflect cryptic circulation of all previously circulating $\mathrm{A}(\mathrm{H} 1 \mathrm{~N} 1)$ clades into early 2021 . Three independent lineages of clade 6b1.A/187A viruses circulated in West Africa (Ghana, Nigeria, and Togo) during 2020, along with a few 6b1.A/156K and 6b1.A/183P-5a viruses (Figure 4 and Extended Data Figure 3). Since May 2021, clade 6b1.A/156K viruses were primarily detected in India, while the other A(H1N1) clades in circulation have been detected sporadically around the world (Figure 4).

An ongoing B/Victoria epidemic in China accounts for the majority of all seasonal influenza viruses detected in 2020/2021 (Extended Data Table 1). Since November 2020, China has consistently detected over 400 B/Victoria cases per week. While B/Victoria clades V1A.1 and 


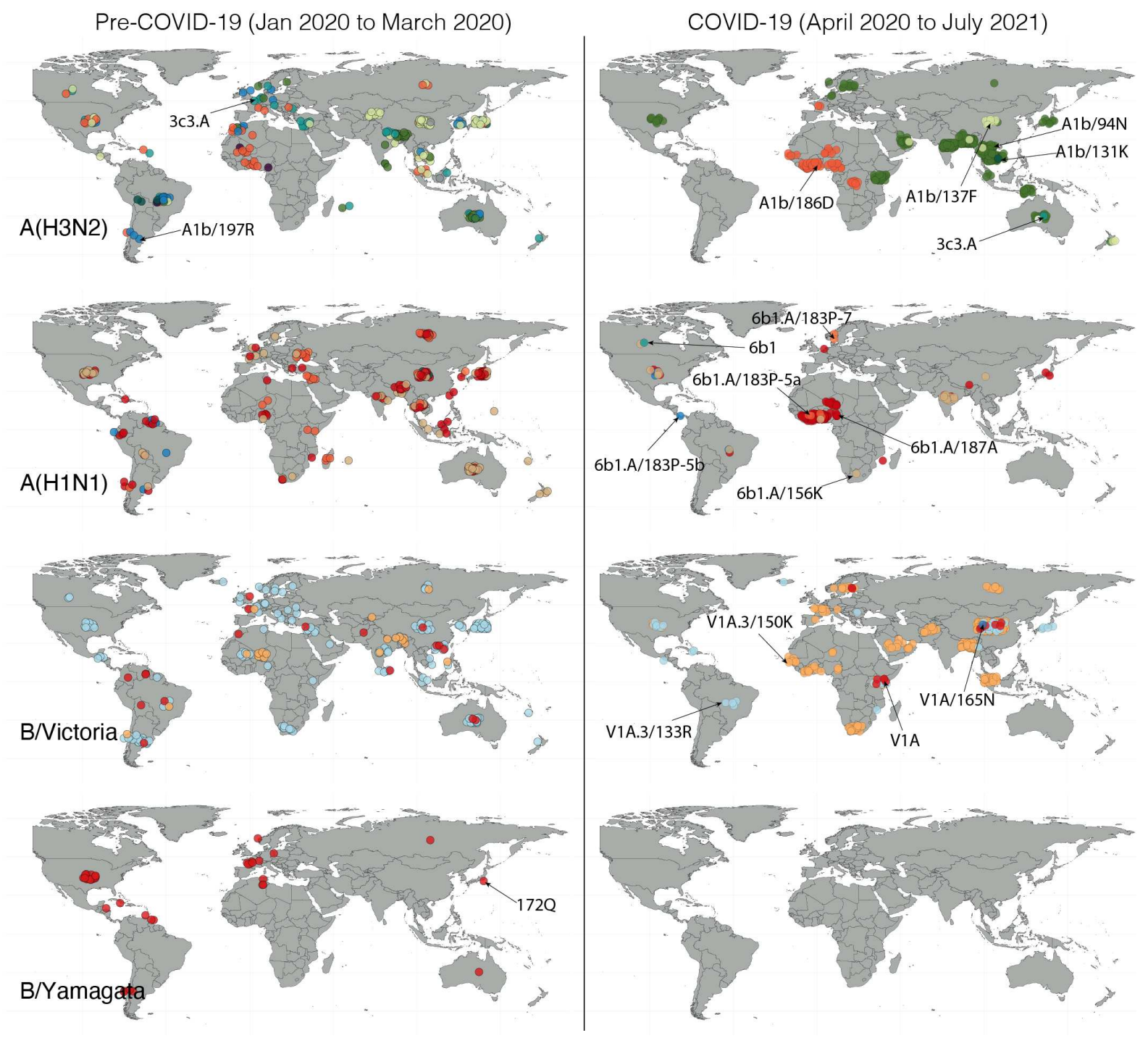

Figure 4. Geographic distribution of HA sequences before (left) and after (right) COVID-

19 emergence. From April 2020 to July 2021, A(H3N2) (590 sequences from 32 countries), $\mathrm{A}(\mathrm{H} 1 \mathrm{~N} 1)$ (254 sequences from 18 countries), and B/Victoria (834 sequences from 34 countries) were available for analysis. In comparison to the 16-month period before April 2020 (December 2018 to March 2020) there was reduction in sequencing of 97\% for A(H3N2), 99\% for $\mathrm{A}(\mathrm{H} 1 \mathrm{~N} 1), 92 \%$ for B/Victoria, and $100 \%$ for B/Yamagata. Last update on August $24^{\text {th }}$, 2021; for an interactive visualization of current seasonal influenza circulation, we refer the reader to the Nextstrain ${ }^{35}$ platform.

V1A.2 have not been reported for over 16 months, five other B/Victoria clades (V1A, V1A.3, V1A.3/133R, V1A.3/150K, and V1A/165N) continue to circulate (Figure 3). Phylogenetic analysis shows the circulation of two distinct lineages of clade V1A.3/150K since late 2020 (Extended Data Figure 4). Initially detected in China in September 2019, one V1A.3/150K lineage has been intermittently detected in other parts of the world up to March 2020. The other 
circulating lineage of clade V1A.3/150K has been detected since late 2020 in parts of China, West Africa, the Middle East, Europe, Russia, Bangladesh, and most recently in Singapore during June 2021 (Figure 4 and Extended Data Table 2). Several highly similar HA sequences were detected in West Africa, the Middle East, and Europe, suggesting limited intercontinental transmission of V1A.3/150K viruses has resumed (Extended Data Figure 4). The B/Victoria clade that was dominant prior to March 2020 globally, V1A.3/133R, was infrequently detected from April 2020 to May 2021, mainly from China, Kenya, the United States, Brazil, and Japan (Figure 4 and Extended Data Table 2). Similarly, clade V1A viruses, which circulated globally in 2019, were only detected in China, Kenya, and Sweden since April 2020 (Extended Data Table 2). Clade V1A/165N has been reported infrequently since 2017, and the most recent sequence was detected in Zhejiang province in China on March 2021, suggesting low level circulation of this B/Victoria clade. In summary, V1A.3/150K has been detected globally in recent months and will likely continue to dominate while other B/Victoria clades (e.g., V1A, V1A.3/133R, V1A/165N) have been regionally detected, albeit at lower frequencies, since April 2020 and may eventually be replaced by descendants of the apparently fitter V1A.3/150K viruses.

Notably, few B/Yamagata detections and no B/Yamagata sequences have been reported since March 2020, which suggests transmission of B/Yamagata has not been sustained and the few cases detected are likely to be false positives. ${ }^{34}$

\section{Transmission dynamics and geographic hotspots of seasonal influenza virus circulation}

We generated large-scale phylogenetic trees of HA sequence data from 2018-2021 to determine the number of independent lineages that originated from viruses circulating during March 2020. Over 50 residual transmission lineages were detected in both IAV and B/Victoria (52\% singleton sequences), a majority of which occurred amongst the major clades described above (A(H3N2) A1b/94, A(H1N1) 6b1.A/187A, and B/Victoria V1A.3/150K) (Figure 5). Most residual transmission lineages were derived from viruses circulating within the same country, province, or geographic region (Figure 4, Extended Data Figures 1-4, and Extended Data Table 3). These results affirm the lack of global dissemination of seasonal influenza viruses during the COVID-19 pandemic and reveal smaller regions with high population densities that can independently sustain influenza virus transmission lineages for extended periods. Furthermore, while detection of $\mathrm{A}(\mathrm{H} 3 \mathrm{~N} 2)$ and $\mathrm{B} /$ Victoria viruses derived 


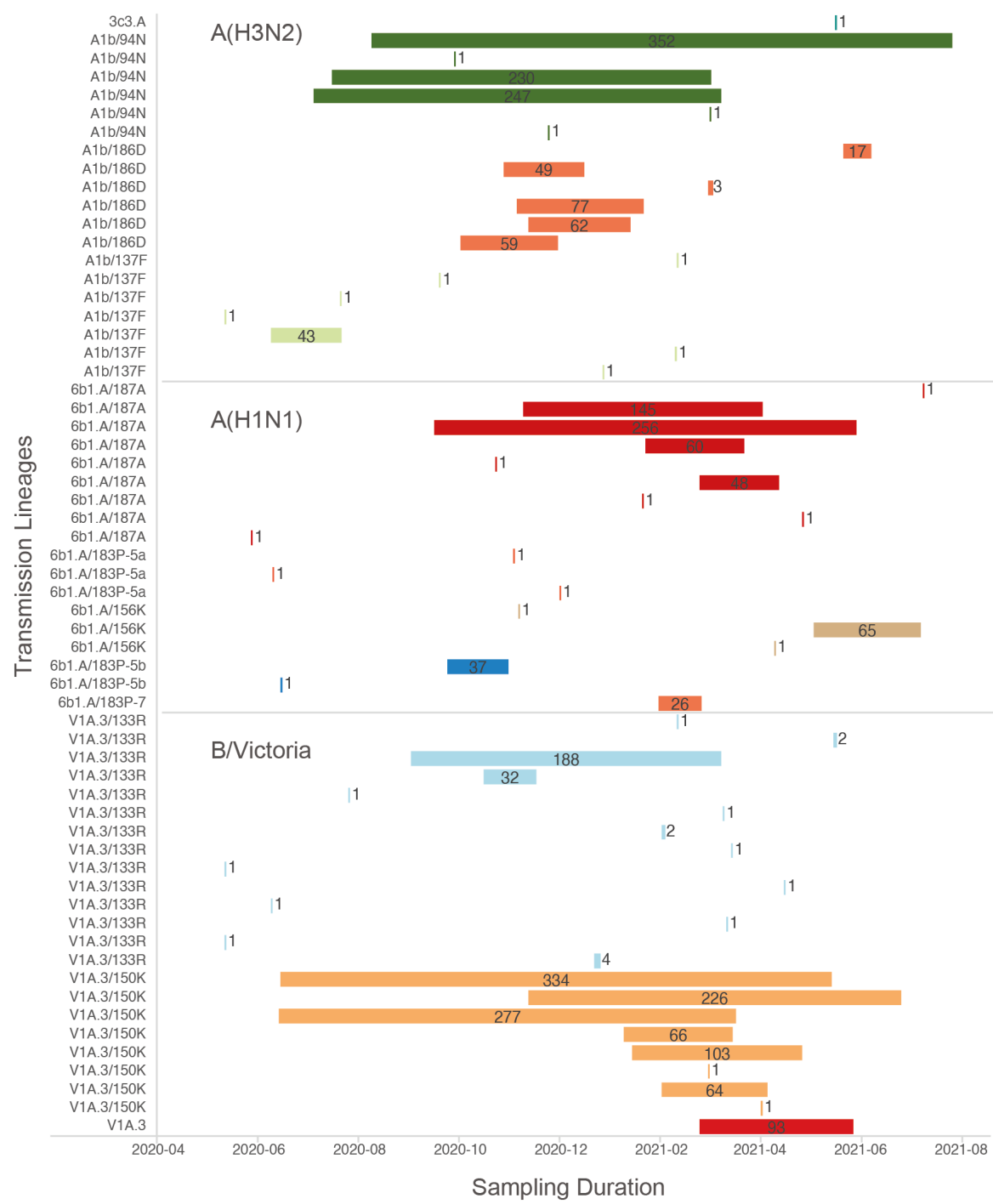

225 Figure 5. Cluster size and duration of influenza transmission lineages that originated 226 before the COVID-19 pandemic. Each colored bar represents a monophyletic transmission 227 lineage inferred from maximum-likelihood phylogenetic analyses of seasonal influenza HA gene sequences in GISAID (see Online Methods). Labels indicate sequence counts per transmission lineage.

230 from pre-COVID-19 influenza virus lineages increased during the first two months of 2021, 231 new detections have since decreased, suggesting many residual transmission chains may have 232 terminated at the end of the 2020/2021 Northern Hemisphere season. Two major lineages 233 continue to be detected in mid-2021, B/Victoria V1A.3/150K was most prevalent, and to a 234 lesser extent, A(H3N2) A1b/94N (Extended Data Table 2 and Extended Data Figures 2,4). 
$\mathrm{A}(\mathrm{H} 1 \mathrm{~N} 1), \mathrm{A}(\mathrm{H} 3 \mathrm{~N} 2)$, and B/Victoria viruses detected during late 2020-early 2021 largely circulated in West and Central Africa. These outbreak reports are supported by genetic sequences, and many of the observed transmission lineages have been regionally maintained since before COVID-19. According to the COVID-19 control policies of individual countries, ${ }^{36}$ internal movement restrictions were enacted across all countries in West Africa by April $13^{\text {th }}$, 2020, and international travel restrictions were in place across the region by March $30^{\text {th }}, 2020$ (Extended Data Figure 5). However, by late July 2020, most countries in West Africa had lifted domestic travel restrictions, and by August 2020 international travel was allowed with some restrictions. A(H3N2) HA gene phylogenies suggest these control measures effectively restricted cross-border spread of influenza viruses, because sequences from Cameroon, Niger, Nigeria, and the Democratic Republic of Congo to January 2021 cluster by country, suggesting containment of virus circulation within each country. By contrast, most Southeast Asian nations maintained relatively stringent domestic and international travel restrictions during 2020/2021, except for Laos and Cambodia, where COVID-19 suppression was followed by A(H3N2) influenza virus outbreaks in 2020. While travel measures impacted international influenza virus migration patterns, we found no correlation between the stringency of public health interventions and domestic transmission of influenza (Extended Data Figure 6).

\section{Discussion}

Despite a transient reduction in influenza surveillance at the start of the COVID-19 pandemic, data generated since then reveal a substantial reduction in global influenza virus circulation under COVID-19 control measures. Lack of exposure to influenza will lower population immunity and increase the severity of large epidemics upon future global resurgence. Notably, countries in North America and Europe with strong influenza surveillance have only sporadically reported the influenza viruses in circulation, including several that have caused outbreaks in Africa and Asia, and B/Yamagata lineage viruses appear to have become extinct around mid-2020.

Roughly one-quarter or more of seasonal influenza cases are caused by IBVs, ${ }^{37}$ and in recent decades the two IBV lineages have caused comparable proportions of influenza cases. Historically, B/Yamagata viruses have caused a greater rate of infection in temperate regions and have infected adults at a greater rate than children, whereas B/Victoria viruses have 
elimination on the evolutionary dynamics of IBV is uncertain. As IBV lineages offer crossprotection, ${ }^{39,40}$ the extinction of $\mathrm{B} /$ Yamagata will leave a higher proportion of individuals susceptible to IBV, enabling faster B/Victoria antigenic evolution. B/Yamagata extinction also creates a vacancy in quadrivalent vaccine formulations, enabling the inclusion of co-circulating clades, which could potentially increase the efficacy of quadrivalent influenza vaccines. It is important to note, the threat of re-introduction of apparently extinct influenza virus lineages could still pose a risk in coming years, as happened with the reemergence of $\mathrm{A}(\mathrm{H} 1 \mathrm{~N} 1)$ in $1977^{41}$ following a 19-year hiatus since the 1958 A(H2N2) pandemic (Figure 6). If B/Yamagata does not reemerge in the next year or so, it may need to be treated as a high consequence pathogen to prevent reintroduction, similar to $\mathrm{A}(\mathrm{H} 2 \mathrm{~N} 2)$ viruses which have not circulated since 1968 and are now held and handled in the higher level BSL-3 laboratory security levels. ${ }^{42}$ Future B/Yamagata positive samples will require urgent confirmation and characterization to be able to better determine the mechanisms that could sustain such low levels of virus circulation - for example, immunocompromised individuals can carry infection for several weeks or months and potentially accumulate additional mutations ${ }^{43-45}$ - or to rule out the possibility that these were in fact false positive test results.

$\begin{array}{lllllllllll}1920 & 1930 & 1940 & 1950 & 1960 & 1970 & 1980 & 1990 & 2000 & 2010 & 2020\end{array}$

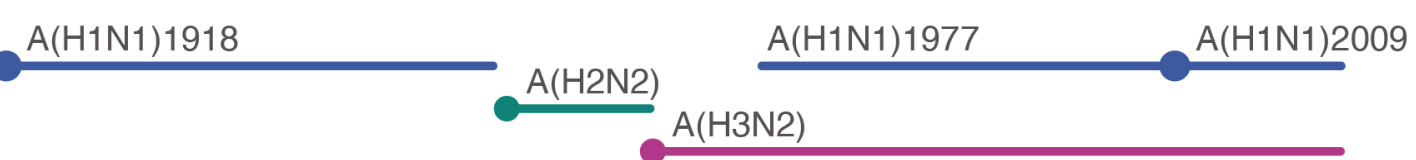

B/Victoria

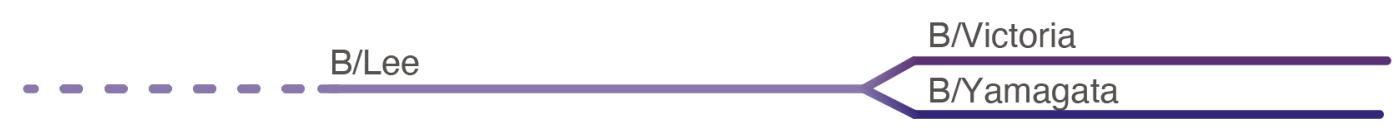

Figure 6. Historical circulation of influenza viruses in the last century. Dots indicate emergence of pandemic strains that replaced previously circulating influenza viruses.

Although two IAV subtypes and two IBV lineages have co-circulated in recent decades, prior to the re-emergence of $\mathrm{A}(\mathrm{H} 1 \mathrm{~N} 1)$ in 1977, only a single IAV subtype and a single IBV lineage circulated among humans (Figure 6). In the early 1980s, IBV diverged from the ancestral $\mathrm{B} /$ Lee lineage into two antigenically distinct lineages. ${ }^{46}$ The survival of two IBV lineages is attributed to the geographic isolation of B/Victoria in China in the 1990s, followed by a global resurgence during 2000-2002. ${ }^{47}$ The continued endemicity of geographically disparate transmission lineages of $\mathrm{A}(\mathrm{H} 3 \mathrm{~N} 2), \mathrm{A}(\mathrm{H} 1 \mathrm{~N} 1)$, and $\mathrm{B} /$ Victoria (compounded by limited availability of clinical isolates) confounds the accuracy of candidate vaccine virus selection, 
and further accumulation of antigenic changes could lead to long-term co-circulation of antigenically distinct lineages, as occurred for IBV. However, the concomitant reduction in population-level immunity towards seasonal influenza suggests global resurgence of any residual viruses could occur in the future and continued vigilance is required.

The emergence of pandemic influenza $\mathrm{A}(\mathrm{H} 2 \mathrm{~N} 2)$ in $1958, \mathrm{~A}(\mathrm{H} 3 \mathrm{~N} 2)$ virus in 1968 , and $\mathrm{A}(\mathrm{H} 1 \mathrm{~N} 1)$ in 2009 from animal reservoirs resulted in the rapid and complete elimination of previously circulating seasonal influenza subtypes (Figure 6). Heterosubtypic cross-protection and competition likely played an important role in eliminating previously circulating viruses during new influenza pandemics. Following the emergence of the 2009 A(H1N1) pandemic, considerably lower levels of A(H3N2) and IBV lineages were detected in 2009 and 2010 globally. However, both non-pandemic viruses sustained transmission throughout the pandemic, and by late 2009 IBVs were detected in considerable numbers in several regions. By 2011, seasonality had resumed with all four viruses being detected, though $A(H 3 N 2)$ evolutionary patterns were significantly altered following co-circulation with 2009 A(H1N1) viruses. ${ }^{32}$ We speculate that heterogeneity in COVID-19 vaccination rates and NPI control policies during 2021/2022 will likely slow the global resurgence of individual influenza lineages, thereby delaying the potential for competition among existing lineages. Upcoming influenza seasons could therefore be compounded in severity as immunity wanes over time for all age groups. ${ }^{16}$ Moreover, the continued evolution of regionally distinct lineages increases risk that the antigens included in the vaccine will not be representative of the viruses that ultimately circulate, thereby reducing vaccine effectiveness.

A previous analysis of global sequencing data highlighted the propensity for sub-tropical regions in Asia to sustain transmission lineages and act as source populations in the emergence of influenza antigenic variants, but limited sequence and surveillance data were available from Africa at that time. ${ }^{5}$ Surveillance capacity in West Africa has since increased with direct support from the WHO and US CDC. In the context of pandemic disruptions to influenza circulation, surveillance in West Africa highlights the potential importance of this region for sustained transmission of influenza and suggests that this region may play a key role in the circulation and maintenance of seasonal influenza lineages. 
323 Knowledge gained from influenza epidemiology and evolution under COVID-19 epidemic control underscores the importance of heightened vigilance and continued influenza vaccination programs as we emerge from the COVID-19 pandemic, as well as the potential consequences of recent changes in seasonal influenza virus lineage diversity. Based on observed genetic diversity and endemicity of circulating lineages, continued travel restrictions will limit the number of regional introductions, and prolonged pandemic mitigation strategies could further impact future seasonal influenza virus circulation and evolution. Ongoing global COVID-19 vaccination rates indicate that middle-income countries may be sufficiently vaccinated by the start of 2022; thus, continuation of mitigation strategies may become impractical, and global travel could return to pre-COVID-19 levels in the near future. As international travel is important for sustaining seasonal influenza transmission, ${ }^{33,48}$ genomic surveillance at border crossings could monitor importation from regions that maintain endemic circulation of seasonal influenza. As illustrated by influenza sequence and surveillance data from 2020 and 2021, East, South, and Southeast Asia have sustained A(H3N2) and B/Victoria transmission lineages, and West Africa has maintained A(H1N1) circulation.

While the COVID-19 vaccination needs should be urgently met in many of these regions, we outline several strategies for the containment of regional influenza outbreaks. (i) Improve pediatric influenza vaccination rates; and (ii) recommend influenza vaccination for travelers, especially those travelling to or from regions with influenza activity. (iii) Laboratory or pointof-care antigen testing of travelers for influenza in addition to COVID-19 could be implemented pre-departure and/or on arrival from countries with influenza activity. (iv) Seasonal vaccines should be made available to countries with recurrent or sustained influenza outbreaks, and large-scale or ring vaccination programs could be undertaken. (v) Reduction in vaccine complexity to trivalent or bivalent formulations should be considered ${ }^{49}$ to reduce cost and make vaccines more affordable to LMICs. Alternatively, replacement of B/Yamagata vaccine component with a second $\mathrm{A}(\mathrm{H} 3 \mathrm{~N} 2)$ strain could improve the vaccine effectiveness if multiple antigenically distinct clades co-circulate and benefit can be shown from including two $\mathrm{A}(\mathrm{H} 3 \mathrm{~N} 2)$ vaccine viruses. The uncertainty in future seasonal influenza circulation provides further incentive for rapid advancement of universal influenza vaccines that confer broad protection against multiple IAV or IBV lineages ${ }^{50-52}$ with the potential to alleviate many of the concerns presented in this manuscript. 
355 Epidemiological trends of seasonal influenza-positive cases and samples tested between 356 January 2015 and July 2021 (Figure 2 and Extended Data Figure 6) were inferred from 357 influenza notifications submitted to the WHO Global Influenza Surveillance and Response 358 System (GISRS). ${ }^{7}$ All human seasonal influenza hemagglutinin (HA) sequences collected from 359 December 2018 to July 2021 were downloaded from GISAID (Extended Data Table 4) and 360 aligned by HA subtype/lineage using MAFFT v.7.22. ${ }^{53}$ Preliminary maximum-likelihood 361 phylogenies were estimated with FastTree v.2.1. ${ }^{54}$ Root-to-tip regression analyses of 362 phylogenetic branch lengths and sampling dates were used to control phylogenetic data quality 363 in TempEst v.1.5.3, ${ }^{55}$ and sequences $<900$ nt were excluded. After adding HA reference 364 sequences (recommended vaccine strains from 2010-2021), the final dataset included 15,526 $365 \mathrm{~A}(\mathrm{H} 3 \mathrm{~N} 2), 16,020 \mathrm{~A}(\mathrm{H} 1 \mathrm{~N} 1), 9,743 \mathrm{~B} /$ Victoria, and 1,029 B/Yamagata sequences.

366 Phylogenetic relationships and divergence times of seasonal influenza HA genes were 367 estimated using IQ-TREE v. $2^{56}$ and the least-square dating method. ${ }^{57}$ Large-scale maximum 368 likelihood analyses using all available HA sequence data were generated by FastTree v.2.154 369 with the generalized time reversible nucleotide substitution model. Branch support was 370 assessed by Shimodaira-Hasegawa test, ${ }^{58}$ and lineages were labelled according to WHO clade 371 designations. The R package 'ggstream' v.0.1 was used to map temporal changes in sampling 372 of seasonal influenza clades. 


\section{Acknowledgements}

This study is supported by the National Institute of Allergy and Infectious Diseases, National Institutes of Health, Department of Health and Human Services of the US, under Contract Nos. U01AI151810, HHSN272201400006C, and 75N93021C00016. The Melbourne WHO Collaborating Centre for Reference and Research on Influenza is supported by the Australian Government Department of Health. The authors thank all NICs and laboratories that supply influenza viruses to the WHO Collaborating Centre for Reference and Research on Influenza for analysis, as well as the authors and the originating laboratories responsible for genetic sequence data shared via GISAID. A complete list of GISAID acknowledgements is provided in Extended Data Table 4.

\section{Author Contributions}

D.V. conceived the study. D.V., S.S., K.M.E., A.K., and R.X. performed analysis and designed the Figures. D.V. and K.M.E. wrote the manuscript with contributions from S.S., R.X., S.A.V., B.J.C., and I.G.B. All authors discussed and approved the manuscript.

\section{Competing Interests statement.}

The authors declare no conflicts of interest. 


\section{References.}

1. Petrova, V.N. \& Russell, C.A. The evolution of seasonal influenza viruses. Nat Rev Microbiol 16, 47-60 (2018).

2. Barberis, I., Myles, P., Ault, S.K., Bragazzi, N.L. \& Martini, M. History and evolution of influenza control through vaccination: from the first monovalent vaccine to universal vaccines. J Prev Med Hyg 57, E115-E120 (2016).

3. Iuliano, A.D., et al. Estimates of global seasonal influenza-associated respiratory mortality: a modelling study. Lancet 391, 1285-1300 (2018).

4. Putri, W., Muscatello, D.J., Stockwell, M.S. \& Newall, A.T. Economic burden of seasonal influenza in the United States. Vaccine 36, 3960-3966 (2018).

5. Bedford, T., et al. Global circulation patterns of seasonal influenza viruses vary with antigenic drift. Nature 523, 217-220 (2015).

6. Barrat-Charlaix, P., Huddleston, J., Bedford, T. \& Neher, R.A. Limited Predictability of Amino Acid Substitutions in Seasonal Influenza Viruses. Mol Biol Evol 38, 27672777 (2021).

7. Hay, A.J. \& McCauley, J.W. The WHO global influenza surveillance and response system (GISRS)-A future perspective. Influenza Other Respir Viruses 12, 551-557 (2018).

8. Li, Y., et al. Global patterns in monthly activity of influenza virus, respiratory syncytial virus, parainfluenza virus, and metapneumovirus: a systematic analysis. Lancet Glob Health 7, e1031-e1045 (2019).

9. Tamerius, J.D., et al. Environmental predictors of seasonal influenza epidemics across temperate and tropical climates. PLoS Pathog 9, e1003194 (2013).

10. Rambaut, A., et al. The genomic and epidemiological dynamics of human influenza A virus. Nature 453, 615-619 (2008).

11. Russell, C.A., et al. The global circulation of seasonal influenza A (H3N2) viruses. Science 320, 340-346 (2008).

12. Patterson Ross, Z., et al. Inter-Seasonal Influenza is Characterized by Extended Virus Transmission and Persistence. PLoS Pathog 11, e1004991 (2015).

13. Caini, S., et al. Characteristics of seasonal influenza A and B in Latin America: Influenza surveillance data from ten countries. PLoS One 12, e0174592 (2017).

14. van de Sandt, C.E., et al. Human Influenza A Virus-Specific CD8+ T-Cell Response Is Long-lived. J Infect Dis 212, 81-85 (2015).

15. Kissling, E., et al. I-MOVE multicentre case-control study 2010/11 to 2014/15: Is there within-season waning of influenza type/subtype vaccine effectiveness with increasing time since vaccination? Euro Surveill 21(2016).

16. Woolthuis, R.G., Wallinga, J. \& van Boven, M. Variation in loss of immunity shapes influenza epidemics and the impact of vaccination. BMC Infect Dis 17, 632 (2017).

17. Bahl, J., et al. Temporally structured metapopulation dynamics and persistence of influenza A H3N2 virus in humans. Proc Natl Acad Sci U S A 108, 19359-19364 (2011).

18. Sullivan, S.G., et al. Where has all the influenza gone? The impact of COVID-19 on the circulation of influenza and other respiratory viruses, Australia, March to September 2020. Euro Surveill 25(2020).

19. Olsen, S.J., et al. Decreased Influenza Activity During the COVID-19 Pandemic United States, Australia, Chile, and South Africa, 2020. MMWR Morb Mortal Wkly Rep 69, 1305-1309 (2020). 
20. Baker, R.E., et al. The impact of COVID-19 nonpharmaceutical interventions on the future dynamics of endemic infections. Proc Natl Acad Sci U S A 117, 30547-30553 (2020).

21. Leung, N.H.L., et al. Respiratory virus shedding in exhaled breath and efficacy of face masks. Nat Med 26, 676-680 (2020).

22. Tang, J.W., et al. Where have all the viruses gone? Disappearance of seasonal respiratory viruses during the COVID-19 pandemic. J Med Virol 93, 4099-4101 (2021).

23. Gomez, G.B., Mahe, C. \& Chaves, S.S. Uncertain effects of the pandemic on respiratory viruses. Science 372, 1043-1044 (2021).

24. Vieira, M.C., et al. Lineage-specific protection and immune imprinting shape the age distributions of influenza B cases. Nat Commun 12, 4313 (2021).

25. Gouma, S., et al. Middle-aged individuals may be in a perpetual state of H3N2 influenza virus susceptibility. Nat Commun 11, 4566 (2020).

26. GAVI, WHO \& CEPI. COVID-19 vaccine doses shipped by the COVAX Facility head to Ghana, marking beginning of global rollout. (2021).

27. Olsen, S.J., et al. Decreased influenza activity during the COVID-19 pandemicUnited States, Australia, Chile, and South Africa, 2020. Am J Transplant 20, 36813685 (2020).

28. Huang, Q.S., et al. Impact of the COVID-19 nonpharmaceutical interventions on influenza and other respiratory viral infections in New Zealand. Nat Commun 12, 1001 (2021).

29. Bedford, T., et al. Integrating influenza antigenic dynamics with molecular evolution. Elife 3, e01914 (2014).

30. Smith, D.J., et al. Mapping the antigenic and genetic evolution of influenza virus. Science 305, 371-376 (2004).

31. Barr, I.G., et al. Intense interseasonal influenza outbreaks, Australia, 2018/19. Euro Surveill 24(2019).

32. Yan, L., Neher, R.A. \& Shraiman, B.I. Phylodynamic theory of persistence, extinction and speciation of rapidly adapting pathogens. Elife 8(2019).

33. Vijaykrishna, D., et al. The contrasting phylodynamics of human influenza B viruses. Elife 4, e05055 (2015).

34. Harper, S.A., et al. Seasonal influenza in adults and children--diagnosis, treatment, chemoprophylaxis, and institutional outbreak management: clinical practice guidelines of the Infectious Diseases Society of America. Clin Infect Dis 48, 10031032 (2009).

35. Hadfield, J., et al. Nextstrain: real-time tracking of pathogen evolution. Bioinformatics 34, 4121-4123 (2018).

36. Hale, T., et al. A global panel database of pandemic policies (Oxford COVID-19 Government Response Tracker). Nat Hum Behav (2021).

37. Caini, S., et al. The epidemiological signature of influenza B virus and its B/Victoria and B/Yamagata lineages in the 21st century. PLoS One 14, e0222381 (2019).

38. Nielsen, J., et al. European all-cause excess and influenza-attributable mortality in the 2017/18 season: should the burden of influenza B be reconsidered? Clin Microbiol Infect 25, 1266-1276 (2019).

39. Skowronski, D.M., et al. Cross-lineage influenza B and heterologous influenza A antibody responses in vaccinated mice: immunologic interactions and B/Yamagata dominance. PLoS One 7, e38929 (2012).

40. Liu, Y., et al. Cross-lineage protection by human antibodies binding the influenza B hemagglutinin. Nat Commun 10, 324 (2019). 
41. Rozo, M. \& Gronvall, G.K. The Reemergent 1977 H1N1 Strain and the Gain-ofFunction Debate. mBio 6(2015).

42. CDC. Interim CDC-NIH Recommendation for Raising the Biosafety Level for Laboratory Work Involving Noncontemporary Human Influenza (H2N2) Viruses. (2005).

43. Memoli, M.J., et al. The natural history of influenza infection in the severely immunocompromised vs nonimmunocompromised hosts. Clin Infect Dis 58, 214-224 (2014).

44. Nichols, W.G., Guthrie, K.A., Corey, L. \& Boeckh, M. Influenza infections after hematopoietic stem cell transplantation: risk factors, mortality, and the effect of antiviral therapy. Clin Infect Dis 39, 1300-1306 (2004).

45. Xue, K.S., et al. Parallel evolution of influenza across multiple spatiotemporal scales. Elife 6(2017).

46. Rota, P.A., et al. Cocirculation of two distinct evolutionary lineages of influenza type B virus since 1983. Virology 175, 59-68 (1990).

47. Shaw, M.W., et al. Reappearance and global spread of variants of influenza B/Victoria/2/87 lineage viruses in the 2000-2001 and 2001-2002 seasons. Virology 303, 1-8 (2002).

48. Geoghegan, J.L., et al. Continental synchronicity of human influenza virus epidemics despite climactic variation. PLoS Pathog 14, e1006780 (2018).

49. Hendriks, J., et al. Quadrivalent influenza vaccines in low and middle income countries: Cost-effectiveness, affordability and availability. Vaccine 36, 3993-3997 (2018).

50. Nachbagauer, R., et al. A chimeric hemagglutinin-based universal influenza virus vaccine approach induces broad and long-lasting immunity in a randomized, placebocontrolled phase I trial. Nat Med 27, 106-114 (2021).

51. Sah, P., et al. Future epidemiological and economic impacts of universal influenza vaccines. Proc Natl Acad Sci U S A (2019).

52. Erbelding, E.J., et al. A Universal Influenza Vaccine: The Strategic Plan for the National Institute of Allergy and Infectious Diseases. $J$ Infect Dis 218, 347-354 (2018).

53. Nakamura, T., Yamada, K.D., Tomii, K. \& Katoh, K. Parallelization of MAFFT for large-scale multiple sequence alignments. Bioinformatics 34, 2490-2492 (2018).

54. Price, M.N., Dehal, P.S. \& Arkin, A.P. FastTree: computing large minimum evolution trees with profiles instead of a distance matrix. Mol Biol Evol 26, 1641-1650 (2009).

55. Rambaut, A., Lam, T.T., Max Carvalho, L. \& Pybus, O.G. Exploring the temporal structure of heterochronous sequences using TempEst (formerly Path-O-Gen). Virus Evol 2, vew007 (2016).

56. Minh, B.Q., et al. IQ-TREE 2: New Models and Efficient Methods for Phylogenetic Inference in the Genomic Era. Mol Biol Evol 37, 1530-1534 (2020).

57. To, T.-H., Jung, M., Lycett, S. \& Gascuel, O. Fast Dating Using Least-Squares Criteria and Algorithms. Systematic Biology 65, 82-97 (2015).

58. Shimodaira, H. \& Hasegawa, M. Multiple Comparisons of Log-Likelihoods with Applications to Phylogenetic Inference. Molecular Biology and Evolution 16, 1114$1116(1999)$. 
2 Extended Data Figure 1 | Evolutionary relationships of the HA genes of A(H3N2) A1b/186D 3 clade. Samples collected since April 2020 are colored. Geographic regions with majority of the 4 samples are labelled alongside the tree. The presented tree is a monophyletic lineage that was 5 extracted from a large-scale analysis comprising all available $\mathrm{A}(\mathrm{H} 3 \mathrm{~N} 2)$ virus HA sequences collected since 2018 .

Extended Data Figure 2 | Evolutionary relationships of the HA genes of A(H3N2) A1b/94N clade. Samples collected since April 2020 are colored. Geographic regions with majority of the samples are labelled alongside the tree. The presented tree is a monophyletic lineage that was extracted from a large-scale analysis comprising all available $\mathrm{A}(\mathrm{H} 3 \mathrm{~N} 2)$ virus HA sequences collected since 2018.

Extended Data Figure 3 | Evolutionary relationships of the HA genes of A(H1N1) 6b1A/187A clade. Samples collected since April 2020 are colored. Geographic regions with majority of the samples are labelled alongside the tree. The presented trees are two closely related monophyletic lineages that were pruned from a large-scale analysis comprising all available $\mathrm{A}(\mathrm{H} 1 \mathrm{~N} 1)$ virus HA sequences collected since 2018.

Extended Data Figure $4 \mid$ Evolutionary relationships of the HA genes of B/Victoria V1A.3/150K clade. Samples collected since April 2020 are colored. Geographic regions with majority of the samples are labelled alongside the tree. The presented tree is a monophyletic lineage that was pruned from a large-scale analysis comprising all available B/Victoria virus HA sequences collected since 2018 .

Extended Data Figure 5 | Internal and international movement restrictions enacted by individual countries against COVID-19. Maps were generated in ourworldindata.org/coronavirus based on the Oxford COVID-19 Government Response Tracker (Hale et al. 2020 Nature Human Behaviour).

Extended Data Figure 6 | Lack of correlation between the stringency of COVID-19 restrictions and seasonal influenza reports for fourteen countries in Africa and Asia. Plots show COVID-19 rate per 100,000, influenza rate per 100,000, stringency index and travel restrictions from May $1^{\text {st }}, 2020$ to August $1^{\text {st }}, 2021$. The COVID-19 and influenza rate plots are labelled by country where rates are at least $10 \%$ of the maximum rate across all countries. The dotted line in all panels is the median. Influenza activity is only shown for countries with (i) epidemic 
activity in FluNet supported by sequence in GISAID collected within three weeks of the reported cases; and (ii) to rule out false positive and sporadic cases not indicative of community transmission, a case report rate of at least 0.02 cases per 100,000 population. Stringency measure is an additive of indicators (school and workplace closures, cancellation of public events, restrictions on gatherings, closings of public transport, public information campaigns, stay at home restrictions, restrictions on internal movement, international travel controls, testing policy, contact tracing, face coverings, vaccination policy) rescaled to vary from 0 to 100. International travel restrictions are quantified as, 0 - no measures; 1 - screening; 2 quarantine of arrivals from high-risk regions; 3 - ban on high-risk regions; and 4 - total border closure. COVID-19 activity was sourced from the WHO COVID-19 Dashboard https://covid19.who.int/ and the multi-sourced Johns Hopkins Coronavirus Resource Center https://coronavirus.jhu.edu/.

\section{Extended Data Tables}

Extended Data Table 1 | Comparison of global confirmed cases of seasonal influenza FluNet data in the 16-month period from December 2018 to March 2020 (before the COVID-19 pandemic) against the 16-month period from April 2020 to July 2021 (during the COVID-19 pandemic).

Extended Data Table 2 | Circulating clades of seasonal influenza viruses observed from sequences in GISAID from April 2020 to July 2021.

Extended Data Table 3 | Transmission lineages of seasonal influenza viruses detected during 2020/2021 inferred using large-scale phylogenetic analysis of all HA sequences in GISAID from January 2018 to July 2021.

Extended Data Table 4 | Acknowledgements for influenza HA sequences downloaded from GISAID. 


\section{Supplementary Files}

This is a list of supplementary files associated with this preprint. Click to download.

- ExtendedDataFig1.pdf

- ExtendedDataFig2.pdf

- ExtendedDataFig3.pdf

- ExtendedDataFig4.pdf

- ExtendedDataFig5.pdf

- ExtendedDataFig6.pdf

- ExtendedDataTable1.xlsx

- ExtendedDataTable2.xlsx

- ExtendedDataTable3.xlsx

- ExtendedDataTable4.xIsx 